\title{
THE NEXT LINEAR COLLIDER DAMPING RING COMPLEX*
}

\author{
J. Corlett ${ }^{\dagger}$ D. Atkinson, S. De Santis, N. Hartman, K. Kennedy, D. Li, S. Marks, Y. Minamihara, H. Nishimura, M. \\ Pivi, D. Reavill, R. Rimmer, R. Schlueter, A. Wolski, LBNL, Berkeley, CA, USA \\ S. Anderson, B. McKee, T. Raubenheimer, M. Ross, J. C. Sheppard, SLAC, Stanford, CA, USA
}

\section{Abstract}

We report progress on the design of the Next Linear Collider (NLC) Damping Rings complexes. The purpose of the damping rings is to provide low emittance electron and positron bunch trains to the NLC linacs, at a rate of $120 \mathrm{~Hz}$. As an option to operate at the higher rate of 180 $\mathrm{Hz}$, two $1.98 \mathrm{GeV}$ main damping rings per beam are proposed, and one positron pre-damping ring. The main damping rings store up to $0.8 \mathrm{amp}$ in 3 trains of 190 bunches each and have normalized extracted beam emittances $\gamma \varepsilon_{x}=3 \mathrm{~mm}-\mathrm{mrad}$ and $\gamma \varepsilon_{y}=0.02 \mathrm{~mm}-\mathrm{mrad}$. The optical designs, based on a theoretical minimum emittance lattice (TME), are described, with an analysis of dynamic aperture and non-linear effects. Key subsystems and components are described, including the wiggler, the vacuum systems and photon stop design, and the higherorder-mode damped RF cavities. Impedance and instabilities are discussed.

\section{INTRODUCTION}

The NLC damping ring complexes provide damping of the $\mathrm{e}^{+} / \mathrm{e}^{-}$beams from the sources, producing stable, lowemittance beams which pass on to bunch length compressors and pre-acceleration before entering the main linacs [1]. The main damping rings of both $\mathrm{e}^{+} / \mathrm{e}^{-}$systems are identical. Due to the large emittance of positrons produced from the target, a pre-damping ring is required to reduce the $\mathrm{e}^{+}$beam emittance to be accommodated by the main damping ring acceptance. Each ring will store multiple trains of bunches at once, and a single fully damped bunch train is extracted while a new train from the source is injected. The repetition rate is $120 \mathrm{~Hz}$, although options to operate at $180 \mathrm{~Hz}$ have been explored.

\section{DESIGN}

The damping ring designs use Theoretical Minimum Emittance (TME) arc cells, with a racetrack layout in which damping wigglers, RF systems, feedback systems, a circumference adjustment chicane, and injection/ extraction hardware are located in the long straights. Damping wigglers are required to provide the necessary damping at $1.98 \mathrm{GeV}$, given a $120 \mathrm{~Hz}$ injection/extraction rate and the extracted beam emittance requirement. General discussion of design criteria are given in [2], and details of the current lattice designs in [3]. Principal parameters of the rings are listed in Table 1.

The rings resemble third-generation synchrotron light

\footnotetext{
* Work supported by the US DOE under contract DE-AC0376SF00098 (LBNL), and DE-AC0376SF00098 (SLAC)

†jncorlett@lbl.gov
}

Table 1: Parameters for the Main Damping Ring and Positron Pre-Damping Ring.

\begin{tabular}{|l|c|c|}
\hline & MDR & PPDR \\
\hline Circumference $(\mathrm{m})$ & 299.792 & 218.336 \\
\hline Max. Current $(\mathrm{A})$ & 0.8 & 0.75 \\
\hline Bunch trains $\mathrm{x}$ & $3 \times 190 \times 1.4$ & $2 \times 190 \times 1.4$ \\
Bunches per train $\mathrm{x}$ & & \\
$\begin{array}{l}\text { Bunch separation } \\
(\mathrm{ns})\end{array}$ & & \\
\hline$v_{\mathrm{x}}, v_{\mathrm{y}}, v_{\mathrm{s}}$ & $27.26,11.14$, & $8.91,7.24$, \\
& 0.0035 & 0.031 \\
\hline$\gamma \varepsilon_{\mathrm{x} \text { equilib. }}(\mathrm{mm}-\mathrm{mrad})$ & 2.17 & 103 \\
\hline$\gamma \varepsilon_{\mathrm{x} \text { extract. }}, \gamma \varepsilon_{\mathrm{y} \text { extract. }}$ & $2.18,0.02$ & 114,69 \\
$(\mathrm{~mm}-\mathrm{mrad})$ & & \\
\hline$\sigma_{\varepsilon}(\%), \sigma_{\mathrm{z}}(\mathrm{mm})$ & $0.09,3.6$ & $0.09,7.2$ \\
\hline$\xi_{\mathrm{x} \text { uncorr. }} \xi_{\mathrm{y}}$ uncorr & $-37.12,-28.24$ & $-10.39,-$ \\
\hline$\tau_{\mathrm{x}}, \tau_{\mathrm{y}}, \tau_{\varepsilon}(\mathrm{ms})$ & $4.85,5.09$, & $4.24,5.13$, \\
\hline $\mathrm{U}_{\mathrm{sr}}(\mathrm{kV} / \mathrm{turn})$ & 2.61 & 2.87 \\
\hline$\alpha_{\mathrm{p}}$ & 777 & 561 \\
\hline $\mathrm{V}_{\mathrm{RF}}(\mathrm{MV})$, & $2.95 \times 10^{-4}$ & $7.05 \times 10^{-3}$ \\
\hline Frequency $(\mathrm{MHz})$ & $1.07,714$ & $3.4,714$ \\
\hline Lattice & $36 \mathrm{TME}$ cells & $16 \mathrm{TME}$ cells \\
\hline
\end{tabular}

sources and the B-Factories in that they must store high current beams $(\sim 1 \mathrm{~A})$ while attaining small emittances, low vacuum pressure, and large synchrotron radiation power loading. Many design and technology issues for

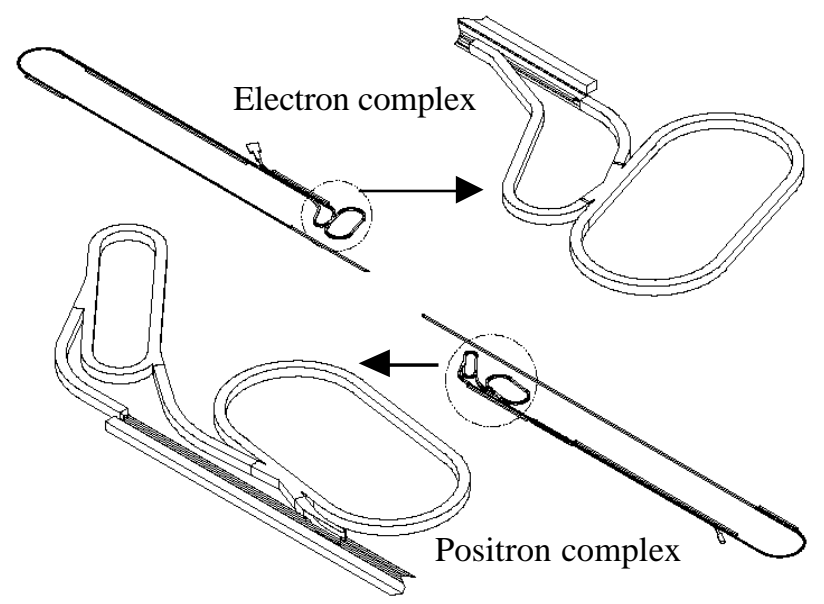

Figure 1. Damping rings layouts, showing $\mathrm{e}^{+}$and $\mathrm{e}^{-}$ complexes at the ends of the main linacs. 


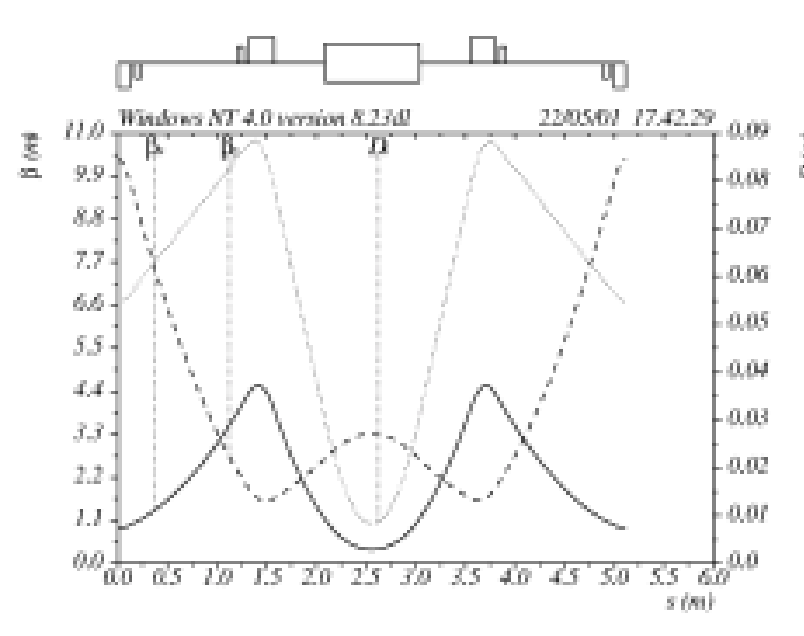

Figure 2. Main ring arc lattice functions.

the damping rings have been successfully demonstrated at operating light sources, and at the B-Factory colliders.

For operational flexibility, the designs allow for an energy range of $\pm 5 \%$, and bunch charge and separation variation of a factor of two such that average current is maintained.

Layout of the damping rings enclosures are shown in Figure 1, in a configuration in which the damping rings complexes are located at the ends of the main linacs. Other configuration options include a central injector complex in which the rings are close to the interaction point and transfer lines connect to the big bends at the ends of the linacs.

Arc dipoles utilise gradient magnets to provide additional focussing, and main ring arc lattice functions are shown in Figure 2. Dynamic aperture is predicted to be in excess of 15 times the injected beam rms transverse dimensions [3]. Obtaining the extracted emittance requires beam-based alignment to determine BPM position relative to the quadrupole centers. Simulations show that extracted beam normalized emittances of $\gamma \varepsilon_{\mathrm{x}} \dagger 3 \mathrm{~mm}$-mrad and $\gamma \varepsilon_{\mathrm{y}}$ $\dagger 0.02$ mm-mrad may be produced with acceptable tolerance on magnet alignment and magnet position control [3]. Skew quadrupoles will be used to correct coupling and minimise residual vertical dispersion.

The damping times of the bare lattice are of the order of $10 \mathrm{~ms}$. To provide sufficiently rapid damping to allow extraction of small emittance beams at $120 \mathrm{~Hz}$, a significant fraction of the circumference is occupied by damping wiggler magnets. The $2.15 \mathrm{~T}, 27 \mathrm{~cm}$ period wiggler has integrated wiggler field of $106.9 \mathrm{~T}^{2} \mathrm{~m}$, providing two-thirds of the total energy loss.

Injection and extraction systems require fast rise and fall times of 65 - $100 \mathrm{~ns}$ to avoid perturbation of stored beams in the ring. Extraction kickers and septa are located after the RF cavities and immediately before the injection hardware, such that the RF system does not experience large transients due to missing bunch trains.
The positron beams from the source have a large edge emittance of $30000 \mathrm{~mm}$-mrad, requiring a pre-damping ring which reduces the emittance by approximately two orders of magnitude. The pre-damping ring lattice is also based on TME arc cells, the acceptance is significantly larger than the main rings and the circumference reduced to accommodate only two trains [3].

\section{TECHNOLOGY}

In addition to the demands of current generation light sources and B-factories, the damping ring complex includes additional challenges associated with fast damping, high throughput injection from a powerful injector, and extraction of stable beams. We have focussed design effort on critical systems including the HOM damped RF cavities, the vacuum systems, and the damping wiggler.

\subsection{RF system}

The RF cavities are higher-order-mode damped singlecell cavities, based on the successful PEP-II design. Three damping waveguides are used to externally load the cavity HOM's, reducing their impedance and thus coupled-bunch growth rates to a level at which feedback systems may control residual instabilities [4,5]. The cavity is designed for a frequency of $714 \mathrm{MHz}$, gap voltage of $500 \mathrm{kV}$ and beam current of $800 \mathrm{~mA}$, and is a conventional reentrant shape except for the addition of the HOM damping ports. Three cavities are sufficient to provide the necessary voltage for the main damping rings, however we require seven cavities to provide the voltage necessary for momentum acceptance in the PPDR.

Designs for the RF window, HOM loads and tuners have been developed. Figure 3 shows a CAD drawing of the cavity; further details may be found in [4].

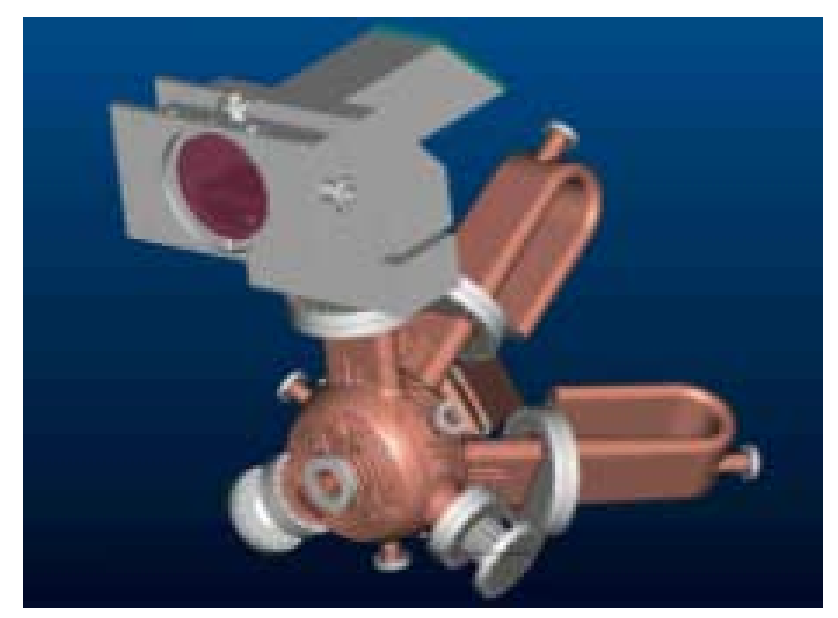

Figure 3. HOM damped RF cavity.

\subsection{Wiggler magnet}

Wigglers are required to provide most of the damping in both the main rings and the pre-ring. The wiggler magnet design is a NdFeB and vanadium permandur hybrid. The 
main ring design has a peak field of $2.15 \mathrm{~T}$, pole gap 2 $\mathrm{cm}$, and period $27 \mathrm{~cm}$. There are ten sections each of length $4.6 \mathrm{~m}$, giving an integrated field-squared of 106.9 $\mathrm{T}^{2} \mathrm{~m}$. The design is similar to insertion devices used on existing synchrotron light sources.

The finite pole width of the wiggler magnet produces nonlinear field components that may deteriorate the dynamic aperture and reduce injection efficiency. The effects on beam dynamics of the field roll-of $\left(B_{y}\right.$ vs $\left.x\right)$ have been investigated and is not believed to be a limiting factor for $13 \mathrm{~cm}$ pole width [6].

The PPDR wiggler has peak field of $2 \mathrm{~T}$, pole gap 4 $\mathrm{cm}$, and period $1 \mathrm{~m}$.

\subsection{Vacuum systems}

The vacuum system in the arcs is based on an aluminum structure with an antechamber in which photon stops and vacuum pumping is located. A narrow slot allows synchrotron radiation to pass from the beam aperture into the antechamber, allows for vacuum conductance to achieve a residual pressure of $10^{-9}$ Torr at the beam, and electromagnetically isolates the beam chamber from the large antechamber to reduce the impedance. Approximately $5.5 \mathrm{~kW}$ of synchrotron radiation power is emitted from each arc dipole, and is absorbed on discrete photon stops, each with a vacuum pump mounted below.

The wiggler occupies $46 \mathrm{~m}$ of the main ring circumference, and produces a synchrotron radiation power of $420 \mathrm{~kW}$. The majority of this power is absorbed on discrete photon stops located between each of the ten wiggler sections, with a vacuum pump mounted below. The aluminum vacuum chamber has a central circular beampipe aperture, with openings along each side to allow synchrotron radiation to pass through to the photon stops. The wiggler vacuum chamber includes facility to install titanium sublimation pumping units along either side of the beamline, as can be seen in figure 4. This distributed pumping along the length of the wiggler is required to maintain a residual pressure of $10^{-9}$ Torr at the beam, given the limited conductance and large surface area of this vacuum chamber. Experiments are under way to determine the feasibility of producing very low outgassing vacuum chambers to eliminate the need for the distributed TSP system [7].

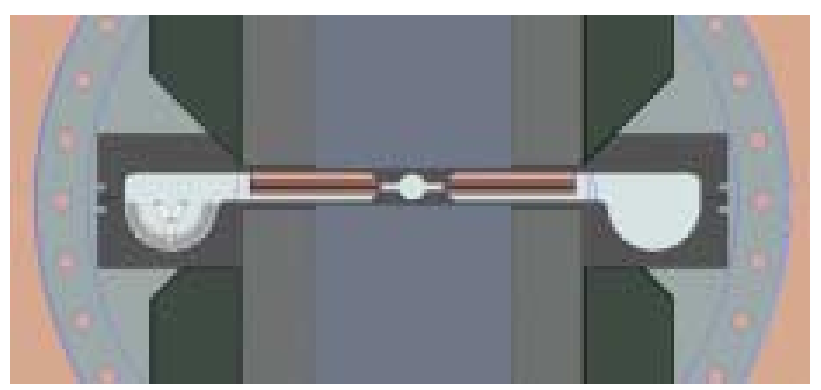

Figure 4. Wiggler section showing vacuum chamber with semi-circular features to hold distributed pumping.
The wiggler photon stops have a contoured surface which is designed to produce a uniform power density of approximately $7 \mathrm{Wmm}^{-2}$ from the incident synchrotron radiation. Mis-steering of $\pm 0.75 \mathrm{~mm}$ on the photon stop results in a still-tolerable maximum power density of 20 $\mathrm{Wmm}^{-2}$. The photon stops are fabricated from Glid-Cop cooled by a serpentine water channel, and the maximum stress is approximately $34,000 \mathrm{psi}$ - a safety factor of two below yield.

\section{IMPEDANCE AND COLLECTIVE EFFECTS}

Wakefields of individual vacuum chamber components have been produced using the 3-D time-domain MAFIA code, and resonant modes have been calculated using MAFIA modelling of the RF cavities.

Calculations of single-bunch effects using the wakefield generated from estimated components designs suggest that instability begins at a bunch population of $1.25 \times 10^{11}$, a factor of eight greater than design value [4,5].

Transverse multi-bunch stability is dominated by the resistive wall impedance, with small contributions from the cavity HOM's. A broad-band, bunch-by-bunch transverse feedback system will be required to maintain beam stability.

Longitudinal coupled-bunch motion may be driven by the fundamental mode of the accelerating cavities, as the cavities are detuned under beamloading. Notch filters in the RF system may be used to damp these few modes.

Simulations of the electron cloud build-up in the positron main ring have been made with the simple geometry of a circular beampipe without antechamber. Corresponding growth rate estimates are less than 10,000 $\mathrm{s}^{-1}$, and the transverse feedback system will damp this motion.

\section{REFERENCES}

[1] J. C. Sheppard et al, "Update on the NLC Injector System Design", this Conference.

[2] M. Ross et al, "The Next Linear Collider Damping Ring Complex", Proc. PAC99, New York, 1999.

[3] A. Wolski and J.N. Corlett, " The Next Linear Collider Damping Ring Lattices", this Conference.

[4] R.A. Rimmer et al, "An RF cavity for the NLC damping rings", this Conference.

[5] J. Corlett et al, "Impedance and instabilities in the NLC damping rings", this Conference.

[6] A. Wolski et al, "Effects of damping wigglers on beam dynamics in the NLC damping rings", this Conference.

[7] S. Shen et al, " Fabrication and measurement of lowoutgassing surfaces for UHV vacuum chambers", this Conference. 